\title{
Novel Macrocyclic Derivatives of Diterpenoid Isosteviol
}

\author{
Olga V. Andreeva, Irina Yu. Strobykina, Olga N. Kataeva, Alexey B. Dobrynin,
} Vasiliy M. Babaev, Ildar Kh. Rizvanov, Daud R. Islamov, and Vladimir E. Kataev@

Dedicated to Academician of the Russian Academy of Sciences A. I. Konovalov on the occasion of his $80^{\text {th }}$ birthday

A.E. Arbuzov Institute of Organic and Physical Chemistry of Kazan Scientific Center of Russian Academy of Sciences, 420088 Kazan, Russia

${ }^{\circledR}$ Corresponding author E-mail: kataev@iopc.ru

\begin{abstract}
A series of novel macrocycles was synthesized on the basis of diterpenoid isosteviol (16-oxo-ent-beyeran-19-oic acid) obtained by acid hydrolysis of the glycosides of the plant Stevia rebaudiana. The macrocycles contain two or four isosteviol moieties connected by diester linkers of various length. Molecular structure of several macrocycles was determined by single crystal X-ray diffraction.
\end{abstract}

Keywords: Macrocyclic compounds, diterpenoids, isosteviol.

\section{Новые макроциклические производные Аитерпеноида изостевиола}

\author{
О. В. Андреева, И. Ю. Стробыкина, О. Н. Катаева, А. Б. Аобрынин, \\ В. М. Бабаев, И. Х. Ризванов, А. Р. Исламов, В. Е. Катаев ${ }^{\circledR}$
}

Посвящается академику РАН А. И. Коновалову по случаю его 80-летнего юбилея

Институт органической и физической химии им. А.Е. Арбузова КазНЦ РАН, 420088 Казань, Россия

${ }^{\circledR}$ E-mail: kataev@iopc.ru

\begin{abstract}
Синтезирована серия новых макроциклов на основе дитерпеноида изостевиола (16-оксо-энт-бейеран-19-овая кислота), полученного кислотным гидролизом гликозидов растения Stevia rebaudiana Bertoni. Maкроцикль содержат два или четыре энт-бейерановых каркаса, соединенных сложноэфирными спейсерами различной длины. Молекулярная структура некоторых соединений определена методом монокристального РСА.
\end{abstract}

Ключевые слова: Макроциклические соединения, дитерпеноиды, изостевиол.

\section{Introduction}

Diterpenoid isosteviol 1 (16-oxo-ent-beyeran-19-oic acid, Figure 1) was known for a long time ago. It was first obtained in 1931, ${ }^{[1]}$ the structure of ent-beyerane oxoacid was assigned to isosteviol in $1955,{ }^{[1 b]}$ and its structure was finally confirmed by X-ray diffraction in 1988. ${ }^{[1 c]}$ Though isosteviol $\mathbf{1}$ has two very reactive groups, the literature from 1955 till 1998 has provided only 6 papers concerned with chemical transformations of this diterpenoid. ${ }^{[1 d-i]}$ Only starting from 1998, isosteviol 1 attracted a great attention of chemists, and a number of papers about its chemical modifications sharply increased. Towards the beginning of 2013, several hundred isosteviol derivatives have been synthesized and described in literature. ${ }^{[2]}$ Recent papers concern with novel diesters, ${ }^{[3 \mathrm{a}]}$ glycosides, ${ }^{[3 \mathrm{~b}]}$ novel nitrogen- 

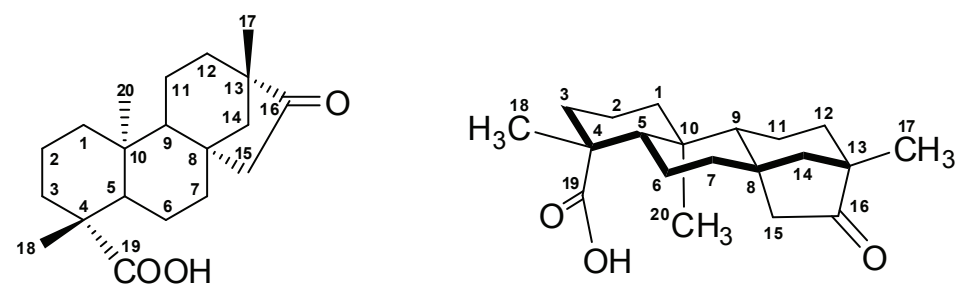

Figure 1. Isosteviol 1.

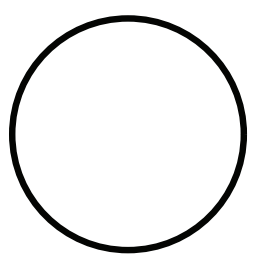

$2 a$

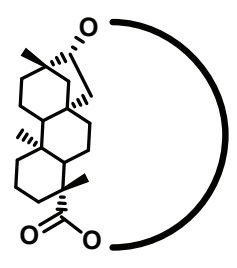

$2 b$

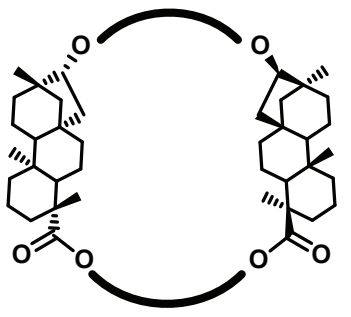

2c

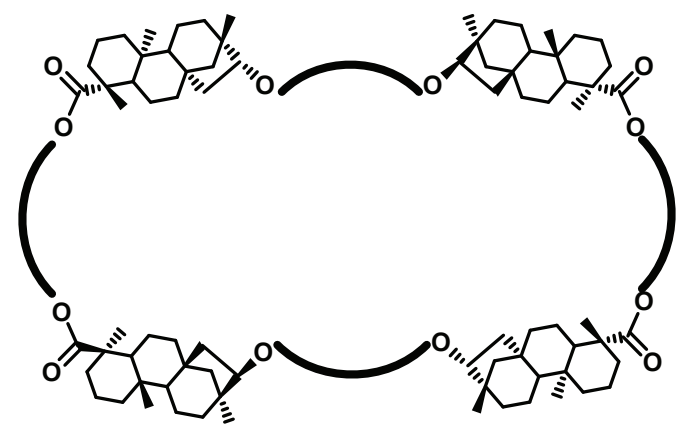

2d

Figure 2. Schematic representation of naturally occurring macrocyclic diterpenoids (2a), and synthetic macrocycles containing one isosteviol molecule (mononuclear macrocycles 2b), two isosteviol molecules (binuclear macrocycles $\mathbf{2 c}$ ), and four isosteviol molecules (tetranuclear macrocycles 2d).

containing heterocyclic derivatives, ${ }^{[3 \mathrm{c}-\mathrm{e}]}$ and the conjugates with thioureas. ${ }^{[3 f, g]}$

Among all isosteviol derivatives macrocycles comprised of one, two and more isosteviol molecules ${ }^{[4 a-e]}$ attract major attention. The matter is that all macrocyclic diterpenoids isolated from higher plants, corals, and algae represent 11to 14-membered macrocycles containing in some cases a fused cyclopropane, cyclopentane or cyclohexane ring. [5a-c] In all these secondary metabolites a macrocycle is a diterpenoid molecule as a whole (Figure 2a). Absolutely different structure of macrocycles containing diterpenoid moieties was implemented on the basis of isosteviol 1. ${ }^{[4 a-e]} \mathrm{A}$ macrocycle was closed around the molecule of this secondary metabolite $^{[4 b]}$ as it is schematically shown in the Figure $2 b$, or coupled two isosteviol molecules ${ }^{[4 a, c-e]}$ (Figure 2c), or even contained four isosteviol molecules ${ }^{[4 \mathrm{e}]}$ (Figure 2d).

The analysis of papers mentioned ${ }^{[4 a-e]}$ shows that systematic investigation of synthetic approach to macrocycles possessing several isosteviol molecules was not carried out. Binuclear macrocycles 2c in which two isosteviol molecules were coupled with each other by diester and anhydride linkers were first obtained as byproducts in 5-7\% yields. ${ }^{[4]}$ The first attempt to synthesize macrocycles comprised of several isosteviol molecules led to complicated mixtures of polynuclear macrocycles which were not separated. ${ }^{[4 \mathrm{~b}]}$ The chloro-anhydride pathway for macrocyclization of isosteviol 1 proposed later afforded binuclear macrocycle of 2c type in which two isosteviol molecules were connected by three diester linkers in $61 \%$ yield. ${ }^{[4]}$ Recently the chloro-anhydride pathway was successfully used for the synthesis of several bi- and tetranuclear macrocycles in which isosteviol molecules were bound by dihydrazide and hydrazone linkers. $\left.{ }^{[4]}\right]$

Herein, we describe our way to bi- and tetranuclear macrocyclic isosteviol derivatives, compare tosylate and chloro-anhydride approaches to the synthesis of such macrocycles, and report the synthesis of several novel macrocyclic isosteviol derivatives.

\section{Experimental}

\section{General}

NMR experiments were carried out with Avance-400 (Bruker) spectrometer in $\mathrm{CDCl}_{3}$ at $400 \mathrm{MHz}$ at $30^{\circ} \mathrm{C}$. High resolution MALDI mass spectra were obtained with a time-of-flight mass spectrometer Ultraflex III TOF/TOF (Bruker Daltonik GmbH, Bremen, Germany) equipped with a Nd:YAG laser and a collision cell. The spectra of positive ions were recorded in a reflectron mode and were processed using the software FlexAnalysis 3.0 (Bruker Daltonik $\mathrm{GmbH}$, Bremen, Germany). Mass accuracy was better than $4 \mathrm{ppm}$. A solutions of analyte samples in $\mathrm{CHCl}_{3}$ at a concentration of $1 \mathrm{mg} / \mathrm{mL}$ were used. Internal calibrations were performed with PEG-1000 or PEG-1500 solutions that mixed with a solutions of analyte samples in the ratio $1: 1 \mathrm{v} / \mathrm{v} .2,5$-Dihydroxybenzoic acid $(5 \mathrm{mg} / \mathrm{mL}$ in methanol) was used as a matrix. The dried-droplet spotting technique was applied in preparing a matrix and analyte solutions. After application of $0.5 \mathrm{~mL}$ of each solution to the target plate MTP AnchorChip ${ }^{\mathrm{TM}}$ (Bruker Daltonik GmbH, Bremen, Germany), a solvent was allowed to evaporate under ambient conditions, and then the plate was inserted into the mass spectrometer for analysis. IR spectra of the compounds synthesized were recorded with Bruker Vector-22 Fourier spectrometer in the wavenumber range from 400 to 4000 $\mathrm{cm}^{-1}$. Melting points of substances were determined on a BOETIUS 
compact heating table. The course of reactions and purity of products were monitored by TLC on Silufol UV-254 (Kavalier, Czech. Rep.) plates. Compounds were detected by $\mathrm{I}_{2}$ vapor. Isolation of individual substances was performed with dry-column flash chromatography on Silicagel KSKG (<0.063 mm, Crom-Lab Ltd, Russia).

All solvents were dried according to standard protocols. Isosteviol 1, dihydroisosteviol 4, diacid 6, and diacid dichloride $\mathbf{2 0}$ were prepared according to the literature. ${ }^{[7,4 c, 8]}$ The physicochemical properties of these compounds agreed with those published.

\section{Syntheses}

Bis(19-nor-4a-chlorocarbonyl-ent-beyeran-16-yl)-hexane1,6-dicarboxilate (19). To a solution of diacid $5(0.2 \mathrm{~g}, 0.26 \mathrm{mmol})$ in dry $\mathrm{CH}_{2} \mathrm{Cl}_{2}(6 \mathrm{~mL})$ was added thionyl chloride $(1 \mathrm{~mL})$, the mixture was refluxed for $6 \mathrm{~h}$ under argon, and the excess thionyl chloride was then removed under reduced pressure. After addition of $\mathrm{CH}_{2} \mathrm{Cl}_{2}$, the mixture was stirred, the solvent was removed under reduced pressure, and the precipitate was dried in vacuo. Yield: $0.21 \mathrm{~g}(100 \%)$. IR (KBr) $v \mathrm{~cm}^{-1}: 1718,1796(\mathrm{C}=\mathrm{O})$.

General procedure for the tosylate pathway synthesis of the macrocycles. A solution of diol ditosylate $(0.25 \mathrm{mmol})$ in $\mathrm{CH}_{3} \mathrm{CN}$ $(15 \mathrm{~mL})$ was added to a solution of diacid 5 (or $\mathbf{6})(0.25 \mathrm{mmol})$ in $\mathrm{CH}_{3} \mathrm{CN}(50 \mathrm{~mL})$ in the presence of $\mathrm{K}_{2} \mathrm{CO}_{3}(0.7 \mathrm{mmol})$ under argon. The mixture was heated for 30-35 h, evaporated, a precipitate was diluted with water and then shaken with chloroform. The organic layer was dried with $\mathrm{MgSO}_{4}$, the solvent was removed under reduced pressure, and then a precipitate was crystallized or purified using chromatography.

General procedure for the chloro-anhydride pathway synthesis of the macrocycles. To the solution of freshly prepared diacid chloride $(0.25 \mathrm{mmol}) \mathbf{1 9}$ (or 20) in $\mathrm{CH}_{2} \mathrm{Cl}_{2}(15 \mathrm{~mL}$ ) triethylamine $(0.07 \mathrm{~mL}, 0.50 \mathrm{mmol})$ and diol solution $(0.25 \mathrm{mmol})$ in $\mathrm{CH}_{2} \mathrm{Cl}_{2}(10$ $\mathrm{mL}$ ) at room temperature under argon were added. The mixture was refluxed for $26 \mathrm{~h}$. After removing the solvent under reduced pressure, a precipitate was purified using chromatography.

2,11,14,23-Tetraoxa-1,12(16,4a)-di(19-nor-ent-beyerana)cyclotetracosaphane-3,10,13,24-tetraone (7). M.p. $139-140{ }^{\circ} \mathrm{C}$ (ethanol). HRMS (MALDI): calcd. for $\mathrm{C}_{56} \mathrm{H}_{88} \mathrm{O}_{8} \mathrm{Na} 911.6371$; found $911.6359[\mathrm{M}+\mathrm{Na}]^{+}$. IR $(\mathrm{KBr}) v \mathrm{~cm}^{-1}: 1720(\mathrm{C}=\mathrm{O}) .{ }^{1} \mathrm{H}$ NMR $(600$ $\left.\mathrm{MHz}, \mathrm{CDCI}_{3}\right) \delta \mathrm{ppm}: 0.70-1.90(\mathrm{~m}, 58 \mathrm{H}$, ent-beyeran skeleton, linker $\left(\mathrm{CH}_{2}\right)_{6}$, linker $\left.\left(\mathrm{CH}_{2}\right)_{4}\right), 0.72\left(\mathrm{~s}, 6 \mathrm{H}, \mathrm{C}^{20} \mathrm{H}_{3}, \mathrm{C}^{20} \mathrm{H}_{3}\right), 0.90(\mathrm{~s}, 6$ $\left.\mathrm{H}, \mathrm{C}^{17} \mathrm{H}_{3}, \mathrm{C}^{17} \mathrm{H}_{3}\right), 1.16\left(\mathrm{~s}, 6 \mathrm{H}, \mathrm{C}^{18} \mathrm{H}_{3}, \mathrm{C}^{18} \mathrm{H}_{3}\right), 2.17(\mathrm{~d}, 2 \mathrm{H}, J=13.4$ $\left.\mathrm{Hz}, \mathrm{C}^{3} \mathrm{H}_{\mathrm{eq}}, \mathrm{C}^{3} \mathrm{H}_{\mathrm{ee}}\right), 2.20-2.37\left(\mathrm{~m}, 4 \mathrm{H}, \mathrm{C}^{16} \mathrm{OC}(\mathrm{O}) \mathrm{CH}_{2}, \mathrm{C}^{16} \mathrm{OC}(\mathrm{O})\right.$ $\left.\mathrm{CH}_{2}\right), 3.95-4.10\left(\mathrm{~m}, 4 \mathrm{H}, \mathrm{C}^{19}(\mathrm{O}) \mathrm{OCH}_{2}, \mathrm{C}^{19^{\prime}}(\mathrm{O}) \mathrm{OCH}_{2}\right), 4.72(\mathrm{dd}, 2$ $\left.\mathrm{H}, J=12.5,6.4 \mathrm{~Hz}, \mathrm{C}^{16} \mathrm{H}, \mathrm{C}^{16} \mathrm{H}\right)$.

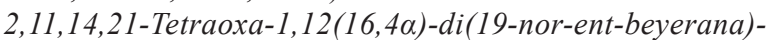
cyclodocosaphane-3,10,13,22-tetraone (8). M.p. 89-90 ${ }^{\circ} \mathrm{C}$ (ethanol). HRMS (MALDI): calcd. for $\mathrm{C}_{54} \mathrm{H}_{84} \mathrm{O}_{8} \mathrm{Na} 883.6058$; found 883.6031 $[\mathrm{M}+\mathrm{Na}]^{+}$. IR $(\mathrm{KBr}) \vee \mathrm{cm}^{-1}: 1723(\mathrm{C}=\mathrm{O}) .{ }^{1} \mathrm{H} \mathrm{NMR}\left(600 \mathrm{MHz}, \mathrm{CDCI}_{3}\right)$ $\delta$ ppm: 0.75- $2.00\left(\mathrm{~m}, 54 \mathrm{H}\right.$, ent-beyeran skeleton, 2 linkers $\left.\left(\mathrm{CH}_{2}\right)_{4}\right)$, $0.72\left(\mathrm{~s}, 6 \mathrm{H}, \mathrm{C}^{20} \mathrm{H}_{3}, \mathrm{C}^{20} \mathrm{H}_{3}\right), 0.90\left(\mathrm{~s}, 6 \mathrm{H}, \mathrm{C}^{17} \mathrm{H}_{3}, \mathrm{C}^{17} \mathrm{H}_{3}\right), 1.15(\mathrm{~s}, 6 \mathrm{H}$, $\left.\mathrm{C}^{18} \mathrm{H}_{3}, \mathrm{C}^{18} \mathrm{H}_{3}\right), 2.17\left(\mathrm{~d}, 2 \mathrm{H}, J=14.4 \mathrm{~Hz}, \mathrm{C}^{3} \mathrm{H}_{\mathrm{eq}}, \mathrm{C}^{3} \mathrm{H}_{\mathrm{eq}}\right), 2.23-2.38$ $\left(\mathrm{m}, 4 \mathrm{H}, \mathrm{C}^{16} \mathrm{OC}(\mathrm{O}) \mathrm{CH}_{2}, \mathrm{C}^{16} \mathrm{OC}(\mathrm{O}) \mathrm{CH}_{2}\right), 3.95-4.06\left(\mathrm{~m}, 4 \mathrm{H}, \mathrm{C}^{19}(\mathrm{O})\right.$ $\left.\mathrm{OCH}_{2}, \mathrm{C}^{19^{\prime}}(\mathrm{O}) \mathrm{OCH}_{2}\right), 4.68$ (dd, $\left.2 \mathrm{H}, J=10.6,4.2 \mathrm{~Hz}, \mathrm{C}^{16} \mathrm{H}, \mathrm{C}^{16^{\prime}} \mathrm{H}\right)$.

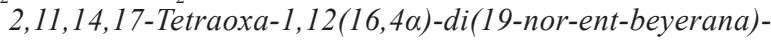
cyclooctadecaphane-3,10,13,18-tetraone (9). M.p. 238-239 ${ }^{\circ} \mathrm{C}$ (hexane). HRMS (MALDI): calcd. for $\mathrm{C}_{50} \mathrm{H}_{76} \mathrm{O}_{8} \mathrm{Na}$ 827.5432; found $827.5400[\mathrm{M}+\mathrm{Na}]^{+}$. IR $(\mathrm{KBr}) \vee \mathrm{cm}^{-1}: 1729(\mathrm{C}=\mathrm{O}) .{ }^{1} \mathrm{H}$ NMR $(600$ $\left.\mathrm{MHz}, \mathrm{CDCI}_{3}\right) \delta$ ppm: 0.70-1.95 (m, $46 \mathrm{H}$, ent-beyeran skeletone, linker $\left.\left(\mathrm{CH}_{2}\right)_{4}\right), 0.72\left(\mathrm{~s}, 6 \mathrm{H}, \mathrm{C}^{20} \mathrm{H}_{3}, \mathrm{C}^{20} \mathrm{H}_{3}\right), 0.91\left(\mathrm{~s}, 6 \mathrm{H}, \mathrm{C}^{17} \mathrm{H}_{3}\right.$, $\left.\mathrm{C}^{17} \mathrm{H}_{3}\right), 1.17$ (s, $\left.6 \mathrm{H}, \mathrm{C}^{18} \mathrm{H}_{3}, \mathrm{C}^{18} \mathrm{H}_{3}\right), 2.18(\mathrm{~d}, 2 \mathrm{H}, J=12.4 \mathrm{~Hz}$, $\mathrm{C}^{3} \mathrm{H}_{\mathrm{eq}}, \mathrm{C}^{3} \mathrm{H}_{\mathrm{eq}}$ ), 2.24-2.37 (m, $\left.4 \mathrm{H}, \mathrm{C}^{16} \mathrm{OC}(\mathrm{O}) \mathrm{CH}_{2}, \mathrm{C}^{16} \mathrm{OC}(\mathrm{O}) \mathrm{CH}_{2}\right)$, $4.13-4.25\left(\mathrm{~m}, 4 \mathrm{H}, \mathrm{C}^{19}(\mathrm{O}) \mathrm{OCH}_{2}, \mathrm{C}^{19}(\mathrm{O}) \mathrm{OCH}_{2}\right), 4.69(\mathrm{dd}, 2 \mathrm{H}, J=$ 10.6, $\left.4.2 \mathrm{~Hz}, \mathrm{C}^{16} \mathrm{H}, \mathrm{C}^{16} \mathrm{H}\right)$.

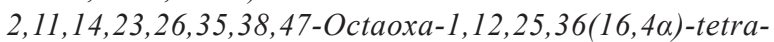
(19-nor-ent-beyerana) cyclooctatetracontaphane-3,10,13,24,27,34, 37,48-octaone (10). M.p. $168-169^{\circ} \mathrm{C}$ (ethanol). HRMS (MALDI): calcd. for $\mathrm{C}_{112} \mathrm{H}_{176} \mathrm{O}_{16} \mathrm{Na}$ 1800.2851; found $1800.2886[\mathrm{M}+\mathrm{Na}]^{+}$. IR $(\mathrm{KBr}) v \mathrm{~cm}^{-1}: 1723(\mathrm{C}=\mathrm{O}) .{ }^{1} \mathrm{H}$ NMR $\left(600 \mathrm{MHz}, \mathrm{CDCI}_{3}\right) \delta \mathrm{ppm}$ : 0.75-1.90 (m, $116 \mathrm{H}$, ent-beyeran skeleton, 2 linkers $\left(\mathrm{CH}_{2}\right)_{6}, 2$ linkers $\left.\left(\mathrm{CH}_{2}\right)_{4}\right), 0.70\left(\mathrm{~s}, 12 \mathrm{H}, \mathrm{C}^{20} \mathrm{H}_{3}, \mathrm{C}^{20} \mathrm{H}_{3}, \mathrm{C}^{20} \mathrm{H}_{3}, \mathrm{C}^{20 "} \mathrm{H}_{3}\right), 0.90$ (s, $\left.12 \mathrm{H}, \mathrm{C}^{17} \mathrm{H}_{3}, \mathrm{C}^{17} \mathrm{H}_{3}, \mathrm{C}^{17^{\prime \prime}} \mathrm{H}_{3}, \mathrm{C}^{17^{\prime \prime}} \mathrm{H}_{3}\right), 1.16\left(\mathrm{~s}, 12 \mathrm{H}, \mathrm{C}^{18} \mathrm{H}_{3}, \mathrm{C}^{18} \mathrm{H}_{3}\right.$, $\left.\mathrm{C}^{18 ”} \mathrm{H}_{3}, \mathrm{C}^{18 ”} \mathrm{H}_{3}\right), 2.16\left(\mathrm{~d}, 4 \mathrm{H}, J=13.6 \mathrm{~Hz}, \mathrm{C}^{3} \mathrm{H}_{\mathrm{eq}}, \mathrm{C}^{3} \mathrm{H}_{\mathrm{e}}, \mathrm{C}^{3} \mathrm{H}_{\mathrm{eq}}\right.$, $\left.\mathrm{C}^{3}{ }^{3} \mathrm{H}_{\mathrm{eq}}\right), 2.30\left(\mathrm{t}, 8 \mathrm{H}, J=7.4 \mathrm{~Hz}, \mathrm{C}^{16} \mathrm{OC}(\mathrm{O}) \mathrm{CH}_{2}, \mathrm{C}^{16} \mathrm{OC}(\mathrm{O}) \mathrm{CH}_{2}\right)$, $\left.\mathrm{C}^{16 "} \mathrm{OC}(\mathrm{O}) \mathrm{CH}_{2}, \mathrm{C}^{16 "} \mathrm{OC}(\mathrm{O}) \mathrm{CH}_{2}\right), 3.90-4.09\left(\mathrm{~m}, 8 \mathrm{H}, \mathrm{C}^{19} \mathrm{OC}(\mathrm{O})\right.$ $\left.\left.\mathrm{CH}_{2}, \mathrm{C}^{19} \mathrm{OC}(\mathrm{O}) \mathrm{CH}_{2}\right), \mathrm{C}^{19} \mathrm{OC}(\mathrm{O}) \mathrm{CH}_{2}, \mathrm{C}^{19}{ }^{9 \prime} \mathrm{OC}(\mathrm{O}) \mathrm{CH}_{2}\right), 4.73$ (dd, $\left.4 \mathrm{H}, J=10.2,4.5 \mathrm{~Hz}, \mathrm{C}^{16} \mathrm{H}, \mathrm{C}^{16} \mathrm{H}, \mathrm{C}^{16 "} \mathrm{H}, \mathrm{C}^{16 "} \mathrm{H}\right)$.

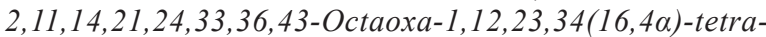
(19-nor-ent-beyerana) cyclotetratetracontaphane-3,10,13,22, 25,32,35,44-octaone (11). M.p. $65-69^{\circ} \mathrm{C}$ (ethanol). HRMS (MALDI): calcd. for $\mathrm{C}_{108} \mathrm{H}_{168} \mathrm{O}_{16} \mathrm{Na}$ 1744.2225; found 1744.2227 $[\mathrm{M}+\mathrm{Na}]^{+}$. IR $(\mathrm{KBr}) \vee \mathrm{cm}^{-1}: 1724(\mathrm{C}=\mathrm{O}) .{ }^{1} \mathrm{H}$ NMR $(600 \mathrm{MHz}$, $\left.\mathrm{CDCI}_{3}\right) \delta$ ppm: 0.79-2.00 (m, $108 \mathrm{H}$, ent-beyeran skeleton, 4 linkers $\left.\left(\mathrm{CH}_{2}\right)_{4}\right], 0.70\left(\mathrm{~s}, 12 \mathrm{H}, \mathrm{C}^{20} \mathrm{H}_{3}, \mathrm{C}^{20} \mathrm{H}_{3}, \mathrm{C}^{20 "} \mathrm{H}_{3}, \mathrm{C}^{20 " \prime} \mathrm{H}_{3}\right), 0.90(\mathrm{~s}, 12 \mathrm{H}$, $\left.\mathrm{C}^{17} \mathrm{H}_{3}, \mathrm{C}^{17} \mathrm{H}_{3}, \mathrm{C}^{17} \mathrm{H}_{3}, \mathrm{C}^{177^{7 \prime} \mathrm{H}_{3}}\right), 1.16\left(\mathrm{~s}, 12 \mathrm{H}, \mathrm{C}^{18} \mathrm{H}_{3}, \mathrm{C}^{18} \mathrm{H}_{3}, \mathrm{C}^{18 ”} \mathrm{H}_{3}\right.$, $\left.\mathrm{C}^{18} 8^{\prime \prime} \mathrm{H}_{3}\right), 2.16\left(\mathrm{~d}, 4 \mathrm{H}, J=12.8 \mathrm{~Hz}, \mathrm{C}^{3} \mathrm{H}_{\mathrm{eq}}, \mathrm{C}^{3} \mathrm{H}_{\mathrm{eq}}, \mathrm{C}^{3 \prime \prime} \mathrm{H}_{\mathrm{eq}}, \mathrm{C}^{3, \prime} \mathrm{H}_{\mathrm{eq}}\right)$, 2.30 (t, $\left.8 \mathrm{H}, J=7.4 \mathrm{~Hz}, \mathrm{C}^{16} \mathrm{OC}(\mathrm{O}) \mathrm{CH}_{2}, \mathrm{C}^{16^{\prime}} \mathrm{OC}(\mathrm{O}) \mathrm{CH}_{2}\right), \mathrm{C}^{16 "} \mathrm{OC}(\mathrm{O})$ $\left.\mathrm{CH}_{2}, \mathrm{C}^{16 "} \mathrm{OC}(\mathrm{O}) \mathrm{CH}_{2}\right), 3.90-4.12\left(\mathrm{~m}, 8 \mathrm{H}, \mathrm{C}^{19} \mathrm{OC}(\mathrm{O}) \mathrm{CH}_{2}, \mathrm{C}^{19} \mathrm{OC}(\mathrm{O})\right.$ $\left.\left.\mathrm{CH}_{2}\right), \mathrm{C}^{19 "} \mathrm{OC}(\mathrm{O}) \mathrm{CH}_{2}, \mathrm{C}^{19 "} \mathrm{OC}(\mathrm{O}) \mathrm{CH}_{2}\right), 4.73(\mathrm{dd}, 4 \mathrm{H}, \mathrm{J}=10.5,5.4$ $\left.\mathrm{Hz}, \mathrm{C}^{16} \mathrm{H}, \mathrm{C}^{16} \mathrm{H}, \mathrm{C}^{16^{2}} \mathrm{H}, \mathrm{C}^{16^{\prime \prime}} \mathrm{H}\right)$.

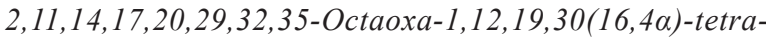
(19-nor-ent-beyerana) cyclohexatriacontaphane-3,10,13,18,21, 28,31,36-octaone (12). M.p. 87-89 ${ }^{\circ} \mathrm{C}$ (hexane). HRMS (MALDI): calcd. for $\mathrm{C}_{100} \mathrm{H}_{152} \mathrm{O}_{16} \mathrm{Na}$ 1632.0973; found $1632.0975[\mathrm{M}+\mathrm{Na}]^{+}$; calcd. for $\mathrm{C}_{100} \mathrm{H}_{152} \mathrm{O}_{16} \mathrm{~K} 1648.0712$; found $1648.0740[\mathrm{M}+\mathrm{K}]^{+}$. IR $(\mathrm{KBr}) v \mathrm{~cm}^{-1}: 1728(\mathrm{C}=\mathrm{O}) .{ }^{1} \mathrm{H}$ NMR $\left(600 \mathrm{MHz}, \mathrm{CDCI}_{3}\right) \delta \mathrm{ppm}$ : 0.75-2.0 (m, $92 \mathrm{H}$, ent-beyeran skeleton, 2 linkers $\left.\left(\mathrm{CH}_{2}\right)_{4}\right), 0.71(\mathrm{~s}$, $\left.12 \mathrm{H}, \mathrm{C}^{20} \mathrm{H}_{3}, \mathrm{C}^{20} \mathrm{H}_{3}, \mathrm{C}^{20} \mathrm{H}_{3}, \mathrm{C}^{20 "} \mathrm{H}_{3}\right), 0.90\left(\mathrm{~s}, 12 \mathrm{H}, \mathrm{C}^{17} \mathrm{H}_{3}, \mathrm{C}^{17^{\prime}} \mathrm{H}_{3}\right.$, $\left.\mathrm{C}^{17 "} \mathrm{H}_{3}, \mathrm{C}^{17^{3} "} \mathrm{H}_{3}\right), 1.17$ (s, $\left.12 \mathrm{H}, \mathrm{C}^{18} \mathrm{H}_{3}, \mathrm{C}^{18} \mathrm{H}_{3}, \mathrm{C}^{18} \mathrm{H}_{3}, \mathrm{C}^{18 " \prime} \mathrm{H}_{3}\right)$, $2.16\left(\mathrm{~d}, 4 \mathrm{H}, J=13.0 \mathrm{~Hz}, \mathrm{C}^{3} \mathrm{H}_{\mathrm{eq}}, \mathrm{C}^{3} \mathrm{H}_{\mathrm{eq}}, \mathrm{C}^{3}{ }^{\prime \prime} \mathrm{H}_{\mathrm{eq}}, \mathrm{C}^{3}{ }^{3 \prime} \mathrm{H}_{\mathrm{eq}}\right), 2.30(\mathrm{t}$, $\left.8 \mathrm{H}, J=7.4 \mathrm{~Hz}, \mathrm{C}^{16} \mathrm{OC}(\mathrm{O}) \mathrm{CH}_{2}, \mathrm{C}^{16} \mathrm{OC}(\mathrm{O}) \mathrm{CH}_{2}\right), \mathrm{C}^{16} \mathrm{OC}(\mathrm{O}) \mathrm{CH}_{2}$, $\left.\mathrm{C}^{16 " ”} \mathrm{OC}(\mathrm{O}) \mathrm{CH}_{2}\right), 4.15-4.29\left(\mathrm{~m}, 8 \mathrm{H}, \mathrm{C}^{19} \mathrm{OC}(\mathrm{O}) \mathrm{CH}_{2}, \mathrm{C}^{19} \mathrm{OC}(\mathrm{O})\right.$ $\left.\left.\mathrm{CH}_{2}\right), \mathrm{C}^{19 "} \mathrm{OC}(\mathrm{O}) \mathrm{CH}_{2}, \mathrm{C}^{19 "} \mathrm{OC}(\mathrm{O}) \mathrm{CH}_{2}\right), 4.74(\mathrm{dd}, 4 \mathrm{H}, \mathrm{J}=10.3,4.2$ $\left.\mathrm{Hz}, \mathrm{C}^{16} \mathrm{H}, \mathrm{C}^{16} \mathrm{H}, \mathrm{C}^{16 "} \mathrm{H}, \mathrm{C}^{16 "} \mathrm{H}\right)$.

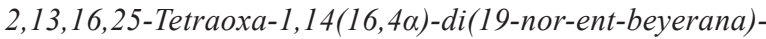
cyclohexacosaphane-3,12,15,26-tetraone (13). M.p. $145-146^{\circ} \mathrm{C}$ HRMS (MALDI): calcd. for $\mathrm{C}_{58} \mathrm{H}_{92} \mathrm{O}_{8} \mathrm{Na}$ 939.6684; found 939.6714 $[M+\mathrm{Na}]^{+}$; calcd. for $\mathrm{C}_{58} \mathrm{H}_{92} \mathrm{O}_{8} \mathrm{~K}$ 955.6424; found $955.6420[M+\mathrm{K}]^{+}$. IR $(\mathrm{KBr}) v \mathrm{~cm}^{-1}: 1728(\mathrm{C}=\mathrm{O}) .{ }^{1} \mathrm{H}$ NMR $\left(600 \mathrm{MHz}, \mathrm{CDCI}_{3}\right) \delta \mathrm{ppm}$ : 0.8-1.9 (m, $62 \mathrm{H}$, ent-beyeran skeleton, 2 linkers $\left.\left(\mathrm{CH}_{2}\right)_{6}\right), 0.71(\mathrm{~s}$, $\left.6 \mathrm{H}, \mathrm{C}^{20} \mathrm{H}_{3}, \mathrm{C}^{20^{\prime}} \mathrm{H}_{3}\right), 0.90\left(\mathrm{~s}, 6 \mathrm{H}, \mathrm{C}^{17} \mathrm{H}_{3}, \mathrm{C}^{17} \mathrm{H}_{3}\right), 1.16\left(\mathrm{~s}, 6 \mathrm{H}, \mathrm{C}^{18} \mathrm{H}_{3}\right.$, $\left.\mathrm{C}^{18^{\prime}} \mathrm{H}_{3}\right), 2.16\left(\mathrm{~d}, 2 \mathrm{H}, J=13.48 \mathrm{~Hz}, \mathrm{C}^{3} \mathrm{H}_{\mathrm{eq}}, \mathrm{C}^{3} \mathrm{H}_{\mathrm{eq}}\right), 2.25-3.34(\mathrm{~m}, 4 \mathrm{H}$, $\left.\mathrm{C}^{16} \mathrm{OC}(\mathrm{O}) \mathrm{CH}_{2}, \mathrm{C}^{16} \mathrm{OC}(\mathrm{O}) \mathrm{CH}_{2}\right), 3.96-4.05\left(\mathrm{~m}, 4 \mathrm{H}, \mathrm{C}^{19} \mathrm{OC}(\mathrm{O}) \mathrm{CH}_{2}\right.$, $\left.\mathrm{C}^{19^{9}} \mathrm{OC}(\mathrm{O}) \mathrm{CH}_{2}\right), 4.71$ (dd, $\left.2 \mathrm{H}, J=10.5,4.1 \mathrm{~Hz}, \mathrm{C}^{16} \mathrm{H}, \mathrm{C}^{16} \mathrm{H}\right)$.

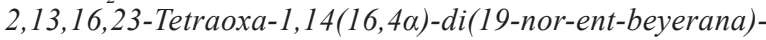
cyclotetracosaphane-3,12,15,24-tetraone (14). M.p. $112-113{ }^{\circ} \mathrm{C}$. HRMS (MALDI): calcd. for $\mathrm{C}_{56} \mathrm{H}_{88} \mathrm{O}_{8} \mathrm{Na}$ 911.6371; found 911.6388 $[M+\mathrm{Na}]^{+}$; calcd. for $\mathrm{C}_{56} \mathrm{H}_{88} \mathrm{O}_{8} \mathrm{~K} 927.6111$; found $927.6096[M+\mathrm{K}]^{+}$. IR $(\mathrm{KBr}) v \mathrm{~cm}^{-1}: 1730(\mathrm{C}=\mathrm{O}) .{ }^{1} \mathrm{H} \mathrm{NMR}\left(600 \mathrm{MHz}, \mathrm{CDCI}_{3}\right) \delta \mathrm{ppm}$ : 0.8-1.95 (m, $58 \mathrm{H}$, ent-beyeran skeleton, linker $\left(\mathrm{CH}_{2}\right)_{4}$, and linker $\left.\left(\mathrm{CH}_{2}\right)_{6}\right), 0.70\left(\mathrm{~s}, 6 \mathrm{H}, \mathrm{C}^{20} \mathrm{H}_{3}, \mathrm{C}^{20} \mathrm{H}_{3}\right), 0.90\left(\mathrm{~s}, 6 \mathrm{H}, \mathrm{C}^{17} \mathrm{H}_{3}, \mathrm{C}^{17} \mathrm{H}_{3}\right)$, $1.15\left(\mathrm{~s}, 6 \mathrm{H}, \mathrm{C}^{18} \mathrm{H}_{3}, \mathrm{C}^{18} \mathrm{H}_{3}\right), 2.17\left(\mathrm{~d}, 2 \mathrm{H}, J=13.02 \mathrm{~Hz}, \mathrm{C}^{3} \mathrm{H}_{\mathrm{eq}}\right.$, $\left.\mathrm{C}^{3} \mathrm{H}_{\mathrm{eq}}\right), 2.23-2.36\left(\mathrm{~m}, 4 \mathrm{H}, \mathrm{C}^{16} \mathrm{OC}(\mathrm{O}) \mathrm{CH}_{2}, \mathrm{C}^{16} \mathrm{OC}(\mathrm{O}) \mathrm{CH}_{2}\right), 3.95-$ $4.07\left(\mathrm{~m}, 4 \mathrm{H}, \mathrm{C}^{19} \mathrm{OC}(\mathrm{O}) \mathrm{CH}_{2}, \mathrm{C}^{19} \mathrm{OC}(\mathrm{O}) \mathrm{CH}_{2}\right), 4.69(\mathrm{dd}, 2 \mathrm{H}, J=$ $\left.10.5,4.2 \mathrm{~Hz}, \mathrm{C}^{16} \mathrm{H}, \mathrm{C}^{16} \mathrm{H}\right)$.

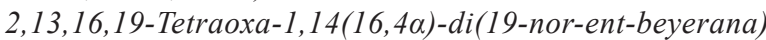
cycloicosaphane-3,12,15,20-tetraone (15). M.p. 202-204 ${ }^{\circ} \mathrm{C}$. HRMS (MALDI): calcd. for $\mathrm{C}_{52} \mathrm{H}_{80} \mathrm{O}_{8} \mathrm{Na} 855.5745$; found 855.5767 $[M+N a]^{+}$; calcd. for $\mathrm{C}_{52} \mathrm{H}_{80} \mathrm{O}_{8} \mathrm{~K} 871.5485$; found $871.5455[M+\mathrm{K}]^{+}$. $\mathrm{IR}(\mathrm{KBr}) v \mathrm{~cm}^{-1}: 1730(\mathrm{C}=\mathrm{O}) .{ }^{1} \mathrm{H} \mathrm{NMR}\left(600 \mathrm{MHz}, \mathrm{CDCI}_{3}\right) \delta \mathrm{ppm}$ : 0.8-1.95 (m, $50 \mathrm{H}$, ent-beyeran skeleton, linker $\left.\left(\mathrm{CH}_{2}\right)_{6}\right), 0.71(\mathrm{~s}$, $\left.6 \mathrm{H}, \mathrm{C}^{20} \mathrm{H}_{3}, \mathrm{C}^{20} \mathrm{H}_{3}\right), 0.90\left(\mathrm{~s}, 6 \mathrm{H}, \mathrm{C}^{17} \mathrm{H}_{3}, \mathrm{C}^{17} \mathrm{H}_{3}\right), 1.16\left(\mathrm{~s}, 6 \mathrm{H}, \mathrm{C}^{18} \mathrm{H}_{3}\right.$, $\left.\mathrm{C}^{18} \mathrm{H}_{3}\right), 2.17\left(\mathrm{~d}, 2 \mathrm{H}, J=13.2 \mathrm{~Hz}, \mathrm{C}^{3} \mathrm{H}_{\mathrm{eq}}, \mathrm{C}^{3} \mathrm{H}_{\mathrm{eq}}\right), 2.23-2.38(\mathrm{~m}, 4 \mathrm{H}$, $\left.\mathrm{C}^{16} \mathrm{OC}(\mathrm{O}) \mathrm{CH}_{2}, \mathrm{C}^{16} \mathrm{OC}(\mathrm{O}) \mathrm{CH}_{2}\right), 4.12-4.23\left(\mathrm{~m}, 4 \mathrm{H}, \mathrm{C}^{19} \mathrm{OC}(\mathrm{O}) \mathrm{CH}_{2}\right.$, $\left.\mathrm{C}^{19} \mathrm{OC}(\mathrm{O}) \mathrm{CH}_{2}\right), 4.70$ (dd, $\left.2 \mathrm{H}, J=10.5,4.2 \mathrm{~Hz}, \mathrm{C}^{16} \mathrm{H}, \mathrm{C}^{16} \mathrm{H}\right)$. 
Table 1. Crystal data and refinement parameters for crystals $6,9,13,15$.

\begin{tabular}{|c|c|c|c|c|}
\hline \multirow{2}{*}{ Parameter } & \multicolumn{4}{|c|}{ Compound } \\
\hline & 6 & 9 & 13 & 15 \\
\hline Empirical formula & $\mathrm{C}_{50} \mathrm{H}_{78} \mathrm{O}_{8}$ & $\mathrm{C}_{50} \mathrm{H}_{76} \mathrm{O}_{8}$ & $\mathrm{C}_{58} \mathrm{H}_{92} \mathrm{O}_{8}$ & $\mathrm{C}_{52} \mathrm{H}_{80} \mathrm{O}_{8}$ \\
\hline Molecular weight & 807.12 & 805.11 & 917.32 & 833.16 \\
\hline Crystal system & Monoclinic & Orthorhombic & Triclinic & Orthorhombic \\
\hline$a / \AA$ & $28.733(8)$ & $13.842(4)$ & $10.866(2)$ & $14.257(3)$ \\
\hline$b / \AA$ & $7.148(2)$ & $14.769(4)$ & $11.525(3)$ & $14.718(3)$ \\
\hline$c / \AA ̊$ & $11.678(3)$ & $22.625(6)$ & $11.647(3)$ & $22.140(5)$ \\
\hline$\alpha / \operatorname{deg}$ & 90.00 & 90.00 & $112.133(2)$ & 90.00 \\
\hline$\beta / \operatorname{deg}$ & 106.866(3) & 90.00 & $91.971(3)$ & 90.00 \\
\hline$\gamma / \operatorname{deg}$ & 90.00 & 90.00 & $94.788(3)$ & 90.00 \\
\hline$V / \AA^{3}$ & 2295.1(11) & $4626(2)$ & $1342.9(5)$ & $4645.7(18)$ \\
\hline$T / \mathrm{K}$ & $296(2)$ & $293(2)$ & $296(2)$ & $296(2)$ \\
\hline Space group & $\mathrm{C} 2$ & P212121 & $\mathrm{P} 1$ & P212121 \\
\hline $\mathrm{Z}$ & 2 & 4 & 1 & 4 \\
\hline Absorption coefficient, $\mu / \mathrm{mm}^{-1}$ & 0.077 & 0.076 & 0.073 & 0.078 \\
\hline$\Theta$ range & $1.99-27.00$ & $2.02-27.00$ & $2.12-27.00$ & $1.84-27.00$ \\
\hline Number of reflections measured & 9320 & 75776 & 18406 & 37242 \\
\hline $\begin{array}{l}\text { Number of independent } \\
\text { reflections }\end{array}$ & 4927 & 10103 & 10955 & 10149 \\
\hline $\begin{array}{l}\text { Number of independent } \\
\text { reflections }(I>2 \sigma)\end{array}$ & 3517 & 4511 & 6859 & 7720 \\
\hline Number of parameters & 277 & 536 & 601 & 547 \\
\hline $\mathrm{R}_{\text {int }}$ & 0.0360 & 0.1061 & 0.0267 & 0.0797 \\
\hline Final R1 values $(\mathrm{I}>2 \sigma(\mathrm{I}))$ & 0.0748 & 0.0690 & 0.0554 & 0.0558 \\
\hline Final $\mathrm{R}_{\mathrm{w}}\left(\mathrm{F}^{2}\right)$ values $(\mathrm{I}>2 \sigma(\mathrm{I}))$ & 0.2052 & 0.1606 & 0.1494 & 0.1331 \\
\hline Final $\mathrm{R}_{1}$ values (all data) & 0.1006 & 0.1610 & 0.0931 & 0.0810 \\
\hline Final $\mathrm{R}_{\mathrm{w}}\left(\mathrm{F}^{2}\right)$ values (all data) & 0.2305 & 0.2071 & 0.1852 & 0.1520 \\
\hline Goodness of fit on $\mathrm{F}^{2}$ & 1.050 & 1.016 & 0.890 & 0.931 \\
\hline
\end{tabular}

\section{X-Ray Crystallography}

The data collection for crystals of compounds $6,9,13,15$ was carried out on a BrukerAXS SMARTAPEXII diffractometer equiped with a graphite-monochromated MoK radiation $(\lambda=0.71073 \AA)$ using the $\omega$ scan mode over the $2 \theta$ range up to $54^{\circ}$. The measurements were performed at room temperature. All data sets were integrated using SAINT ${ }^{[6 a]}$ as implemented in the APEX2 software package. ${ }^{[6 b]}$ Semiempirical absorption correction was carried out using program SADABS. ${ }^{[6 c]}$ The structures were solved by direct methods using SHELXS and refined using SHELXL programs. ${ }^{[6 d]}$ The structural refinement was performed on $\mathrm{F}^{2}$ using all data. All calculations were performed using WINGX ${ }^{[6 e]}$ crystallographic suite of programs. The drawings of molecular structures were prepared using Platon for Windows Taskbar v.1.15. ${ }^{[6]]}$ and Mercury 3.1. ${ }^{[6]]}$ The crystal data and refinement parameters are listed in Table 1. All non-hydrogen atoms were refined anisotropically, the hydrogen atoms being placed in calculated positions and treated as riding on their parent. The linkers of compounds $\mathbf{6}$ and $\mathbf{9}$ are disordered over two positions with occupancy 0.37:0.63 and 0.39:0.61 respectively.

The X-ray crystal structure data for $6,9,13,15$ were deposited in the Cambridge Crystallographic Data Centre (Nos. 975930, 975931, 975932, 975933) as cif-files and can be obtained at the address: www.ccdc.cam.uk/conts/retrieving.html or deposit@ccdc. cam.ac.uk.

\section{Results and Discussion}

Initiating the synthesis of bi- and tetranuclear macrocyclic isosteviol derivatives we considered that exactly binuc-lear isosteviol derivatives 3 (Figure 3) obtained by interaction of dihydroisosteviol 4 with dicarboxylic acids chlorides ${ }^{[9]}$ can be used as suitable starting compounds. The reason is that molecular structures of diacids 3 depend on the length of diester linker bonding two isosteviol fragments (Figure 3). According to the X-ray data, ${ }^{[10]}$ when $n=1$ (or 4), diacid molecules have completely folded tweezer-like structure of 3a kind (Figure 3) with one isosteviol moiety overhanging another. On the contrary, when $n=6$, diacid molecules have completely unfolded structure of $\mathbf{3 b}$ kind (Figure 3 ) in which isosteviol moieties were moved far apart. ${ }^{[10]}$

It was evident for us that a reaction of both diacid having tweezer-like structure 3a and diacid having unfolded 


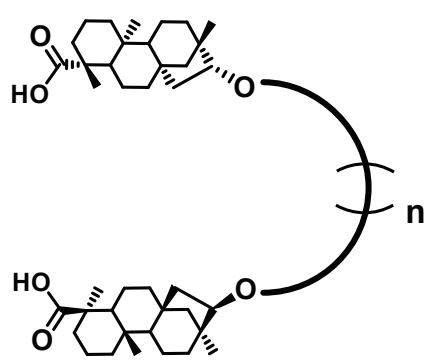

$3 a$

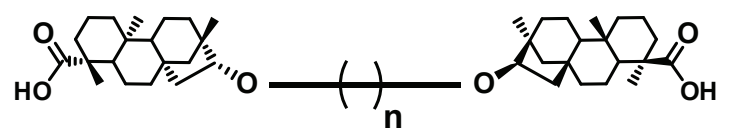

$3 b$

Figure 3. Schematic representation of folded tweezer-like (3a) and unfolded (3b) structures of binuclear isosteviol derivatives. The grey ellipses signify ent-beyerane skeletons.

structure 3b (Figure 3) with a binucleophile having a length which agrees closely with the distance between the $\mathrm{COOH}$ groups of diacids should afford binuclear macrocycles of $\mathbf{2 c}$ kind (Figure 2). We also were sure that a reaction of diacid having unfolded structure $\mathbf{3 b}$ (Figure 3 ) with a binucleophile having the length which is much less than the distance between the $\mathrm{COOH}$ groups of diacid should lead exclusively to a tetranuclear macrocycle of $\mathbf{2 d}$ kind (Figure 2).

To examine this hypothesis we carried out the reactions of diacid $\mathbf{5}^{[9]}$ which, according to X-ray data, ${ }^{[10]}$ has unfolded structure 3b with ditosylates of 1,8-n-octanediol, 1,6- $n$ hexanediol, and ethylene glycol (Scheme 1). To obtain diacid $\mathbf{5}$, firstly isosteviol $\mathbf{1}$ was converted to the dihydroisosteviol 4 by the reaction with $\mathrm{NaBH}_{4}$ in $\mathrm{CH}_{3} \mathrm{OH},{ }^{[8]}$ and compound 4 was obtained in the form of $16(R)$-epimer as in many other cases. [7,8,11a-c] Then dihydroisosteviol 4 was subjected to interaction with suberoyl chloride. In contrast with the known protocol ${ }^{[9]}$ the reaction was carried out in $\mathrm{CH}_{2} \mathrm{Cl}_{2}$ in the presence of pyridine and DMAP that allowed to increase the yield of diacid $\mathbf{5}$ up to $50 \%$. The reaction of diacid 5 with diols ditosylates was carried out following similar procedure as the synthesis of isosteviol esters ${ }^{[12]}$ in $\mathrm{CH}_{3} \mathrm{CN}$ in the presence of $\mathrm{K}_{2} \mathrm{CO}_{3}$ under reflux for 30-35 h. Surprisingly, in all cases, even in the reaction of diacid 5 with ethylene glycol ditosylate, binuclear macrocycles 7-9 appeared to be the main reaction products. They were isolated by chromatography in 23-38 \% yields (Table 2). Tetranuclear macrocycles were formed in low yields just in the reaction of diacid 5 with 1,6-n-hexanediol ditosylate that afforded the macrocycle $\mathbf{1 1}$ in $7 \%$ yield, and in the reaction of diacid 5 with ethylene glycol ditosylate that afforded the macrocycle
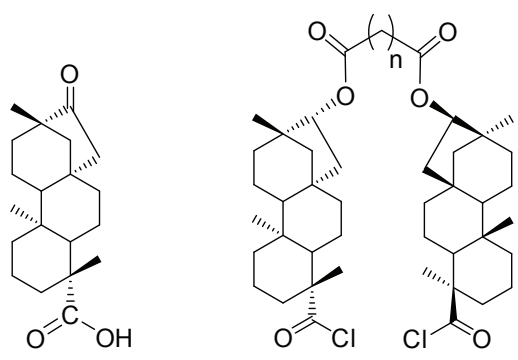

$$
1
$$

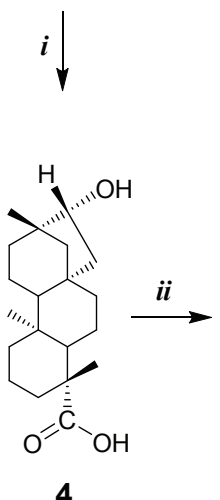

$19(n=6)$

$20(\mathrm{n}=8)$

4

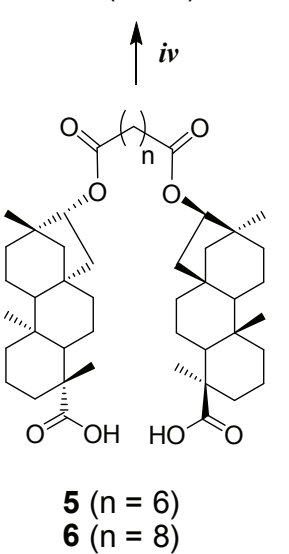

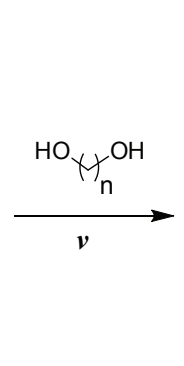

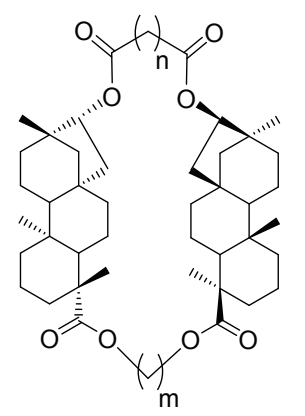

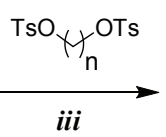

$$
\begin{aligned}
& 7(n=6, m=8) \\
& 8(n=6, m=6) \\
& 9(n=6, m=2) \\
& 13(n=8, m=8) \\
& 14(n=8, m=6) \\
& 15(n=8, m=2)
\end{aligned}
$$

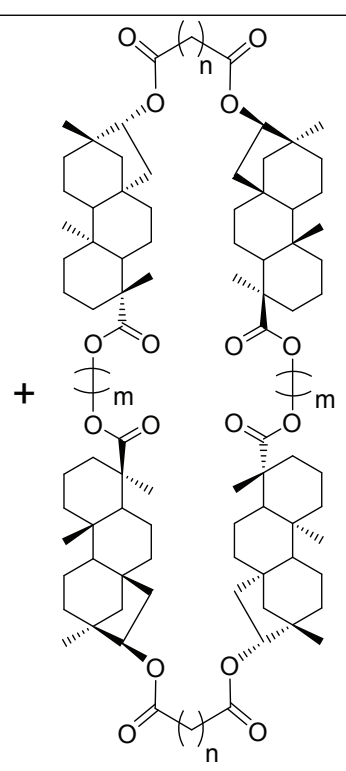

$10(\mathrm{n}=6, \mathrm{~m}=8)$

$11(\mathrm{n}=6, \mathrm{~m}=6)$

$12(n=6, m=2)$

$16(\mathrm{n}=8, \mathrm{~m}=8)$

$17(\mathrm{n}=8, \mathrm{~m}=6)$

$18(\mathrm{n}=8, \mathrm{~m}=2)$

Scheme 1. Reagents and conditions: (i) $\mathrm{NaBH}_{4}, \mathrm{CH}_{3} \mathrm{OH}$, rt; (ii) $\mathrm{Cl}(\mathrm{O}) \mathrm{C}\left(\mathrm{CH}_{2}\right) \mathrm{C}(\mathrm{O}) \mathrm{Cl}, \mathrm{CH}_{2} \mathrm{Cl}_{2}$, DMAP, Py, $\mathrm{Ar}$; (iii) $\mathrm{CH}_{3} \mathrm{CN}_{2} \mathrm{~K}_{2} \mathrm{CO}_{3}$, reflux; (iv) $\mathrm{SOCl}_{2}, \mathrm{CH}_{2} \mathrm{Cl}_{2}, 40^{\circ} \mathrm{C}, 6 \mathrm{~h}$; (v) $\mathrm{HO}\left(\mathrm{CH}_{2}\right)_{\mathrm{n}} \mathrm{OH}, \mathrm{CH}_{2} \mathrm{Cl}_{2}, \mathrm{NEt}_{3}, \mathrm{Ar}, 40^{\circ} \mathrm{C}$. 
Table 2. Yields of binuclear and tetranuclear macrocycles in the reactions of diacids $\mathbf{5}, \mathbf{6}$ with ditosylates (pathway iii), and acid dichlorides $19,20$ with diols (pathway $\mathbf{v})^{a}$

\begin{tabular}{|c|c|c|c|c|c|}
\hline \multicolumn{3}{|c|}{ Binuclear macrocycles } & \multicolumn{3}{|c|}{ Tetranuclear macrocycles } \\
\hline compound & pathway iii & pathway $\mathbf{v}$ & compound & pathway iii & pathway $\mathbf{v}$ \\
\hline 7 & $37 \%$ & $17 \%$ & 10 & n.d. ${ }^{b}$ & $3 \%$ \\
\hline 8 & $23 \%$ & $32 \%$ & 11 & $7 \%$ & $2 \%$ \\
\hline 9 & $38 \%$ & $19 \%$ & 12 & $6 \%$ & $2 \%$ \\
\hline 13 & $86 \%$ & $72 \%$ & 16 & n.d. ${ }^{b}$ & n.d. \\
\hline 14 & $36 \%$ & $44 \%$ & 17 & + & n.d. \\
\hline 15 & $25 \%$ & $27 \%$ & 18 & + & n.d. \\
\hline
\end{tabular}

aPlus sign denotes that a compound was formed in a trace amount registered by MS MALDI.

${ }^{\mathrm{b}} \mathrm{A}$ compound was not detected by MS MALDI.

12 in $6 \%$ yield. The fact that the reaction of diacid 5 having unfolded structure of $\mathbf{3 b}$ kind $^{[10]}$ (Figure 3) with ethylene glycol ditosylate possessing the length that is much less than the distance between the $\mathrm{COOH}$ groups of diacid $\mathbf{5}$ provided mainly the binuclear macrocycle 9, rather than tetranuclear macrocycle $\mathbf{1 2}$ was perfectly incomprehensible. This raised the question: perhaps the reason was that the structure of diacid $\mathbf{5}$ is not unfolded enough? Then we supposed that the increase of the distance between isosteviol moieties in diacid 5 should guarantee much unfolded structure and used diacid $\mathbf{6}$ (Scheme 1) as a starting compound for the macrocyclization because the diester linker binding isosteviol moieties in acid 6 is by two $\mathrm{CH}_{2}$ units longer than the diester linker in acid $\mathbf{5}$.

Results obtained seemed to be unexpected. The reactions of diacid $\mathbf{6}$ with those ditosylates as in the case of diacid $\mathbf{5}$ under the same conditions led exclusively to binuclear macrocycles of 2c kind (Table 2). Tetranuclear macrocycles of $\mathbf{2 d}$ kind (Figure 2) were observed only in trace amounts (Table 2). Thus, the reaction of diacid $\mathbf{6}$ with ditosylate of 1,8- $n$-octanediol afforded binuclear macrocycle 13 in excellent yield (86\%). The tetranuclear macrocycle 16 was not observed. The reactions of diacid $\mathbf{6}$ with ditosylates of 1,6- $n$-hexanediol and ethylene glycole afforded binuclear macrocycles 14 and 15 in good yields (36\% and $25 \%$ respectively). Tetranuclear macrocycles $\mathbf{1 7}$ and $\mathbf{1 8}$ were observed by MS MALDI only in trace amounts.

Anyhow, reactions of diacid $\mathbf{5}$ and $\mathbf{6}$ with diols ditosylates provided macrocyclic derivatives in good yields (Table 2). According to MS MALDI data, unreacted starting compounds and various by-products (derivatives of diacids 5, 6 as well as unclosed precursors of tetranuclear macrocycles 10-12, 16-18 having $\omega$-hydroxyalkyl and $\omega$-TsO-alkyl moieties) were presented in all reaction mixtures along with target macrocycles. We assumed that the TsO group is not highly reactive when interacting with isosteviol carboxyl group shielded by bulky $\mathrm{C}^{18} \mathrm{H}_{3}$ and $\mathrm{C}^{20} \mathrm{H}_{3}$ groups, and used so-called chloro-anhydride approach for the synthesis of macrocyclic isosteviol derivatives. For this purpose diacid $\mathbf{6}$ was converted to acid dichloride $\mathbf{2 0}$ according to known protocol. ${ }^{[4 \mathrm{c}]}$ In a similar way acid dichloride $\mathbf{1 9}$ was obtained from diacid $\mathbf{5}$ (Scheme 1). Then the macrocyclization of compounds $\mathbf{1 9}$ and 20 was performed by reactions with $1,8-n$-octanediol, 1,6- $n$ hexanediol, and ethylene glycol in $\mathrm{CH}_{2} \mathrm{Cl}_{2}$ under reflux. The yields presented in Table 1 demonstrate that nothing new was achieved by the change of synthetic pathway to macrocyclic isosteviol derivatives. Likewise, binuclear macrocycles of 2c kind (Figure 2) appeared to be main products but their yields decreased in comparison with those achieved in the course of the tosylate pathway (Table 2). As to tetranuclear macrocycles of $\mathbf{2 d}$ kind (Figure 2), reactions of acid dichloride 19 with diols afforded macrocycles 10-12 in low yields (2-3\%). According to MS MALDI data, reactions of diacid chloride 20 with diols mentioned above did not lead to tetranuclear macrocycles 16$\mathbf{1 8}$ even in trace amounts.

Thus, tosylate and chloro-anhydride pathways of obtaining isosteviol macrocyclic derivatives from diacid 5 and $\mathbf{6}$ led to similar results. Moreover the reason why our hypothesis that a reaction of unfolded acid with a short binucleophile should afford exclusively tetranuclear macrocycles appeared to be a riddle.

The clue to the mystery was found when we have luck in growing the single crystal of diacid $\mathbf{6}$ and carried out X-ray diffraction study. Contrary to our supposition, it was found that diacid 6 possessing more longer linker between isosteviol moieties than diacid $\mathbf{5}$, in contrast with diacid $\mathbf{5}$, has not an unfolded structure of $\mathbf{3 b}$ kind (Figure 3 ) but has the maximum folding, sandwich-like structure presented in Figure 4. It is obvious from Figure 4 that the molecular structure of diacid $\mathbf{6}$ is perfectly predisposed to a macrocyclization by a binucleophile whose length is close to the sebacoyl linker $(n=8)$ connecting isosteviol moieties. That is the reason for the reaction of diacid $\mathbf{6}$ with the ditosylate of 1,8-octanediol to give binuclear macrocycle $\mathbf{1 3}$ in such excellent yield ( $86 \%$ ). According to the X-ray data, macrocycle 13 preserved a sandwich-like structure which is presented in Figure 5. Based on this fact one can suppose that the sandwich-like structure of diacid $\mathbf{6}$ is probably retained when going from the crystalline phase to a greatly diluted reaction solution. In principle this is not unusual case as the literature has provided the example of how one binuclear isosteviol derivative remained tweezer-like structure when going from a crystal to a greatly diluted reaction solution. ${ }^{[13]}$

Probably the sandwich-like structure of diacid $\mathbf{6}$ is so thermodynamically stable in solution that it is still retained in the course of the reaction with ditosylate of ethylene glycol because the molecular structure of macrocycle $\mathbf{1 5}$ also resembles slightly distorted sandwich (Figure 6). The surprising thing was that the reaction of diacid $\mathbf{5}$ having unfolded structure of $\mathbf{3 b}$ type (Figure 3 ) in crystal ${ }^{[10]}$ with the ditosylate of ethylene glycol afforded macrocycle 9 


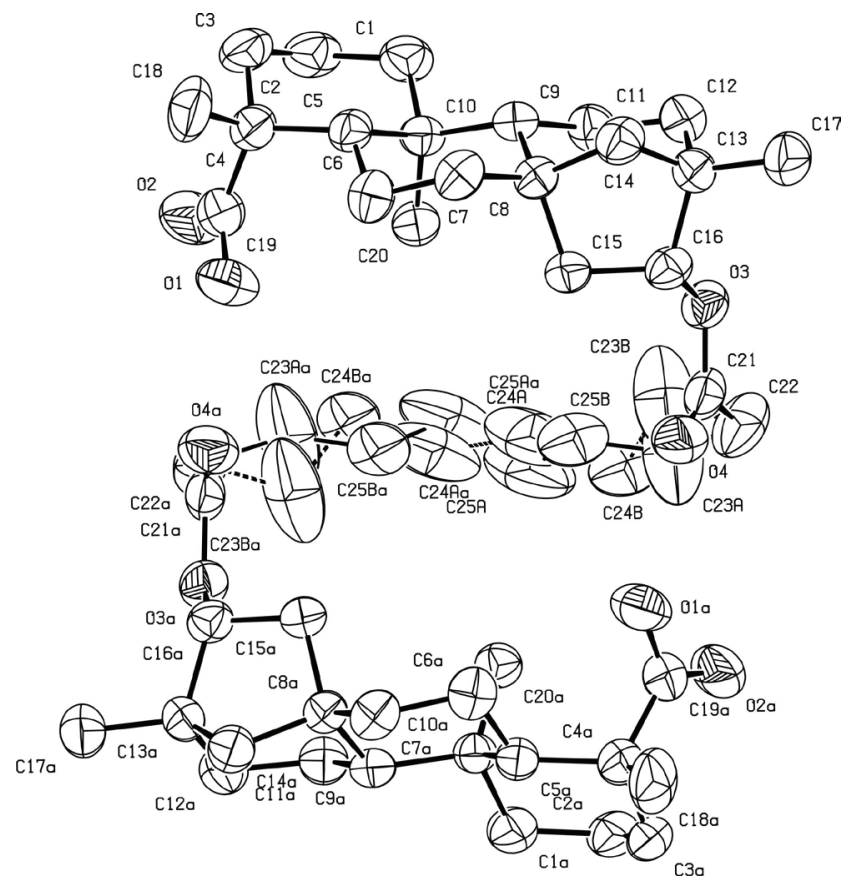

Figure 4. X-ray structure of diacid 6 (ORTEP drawing, hydrogen atoms are not shown).

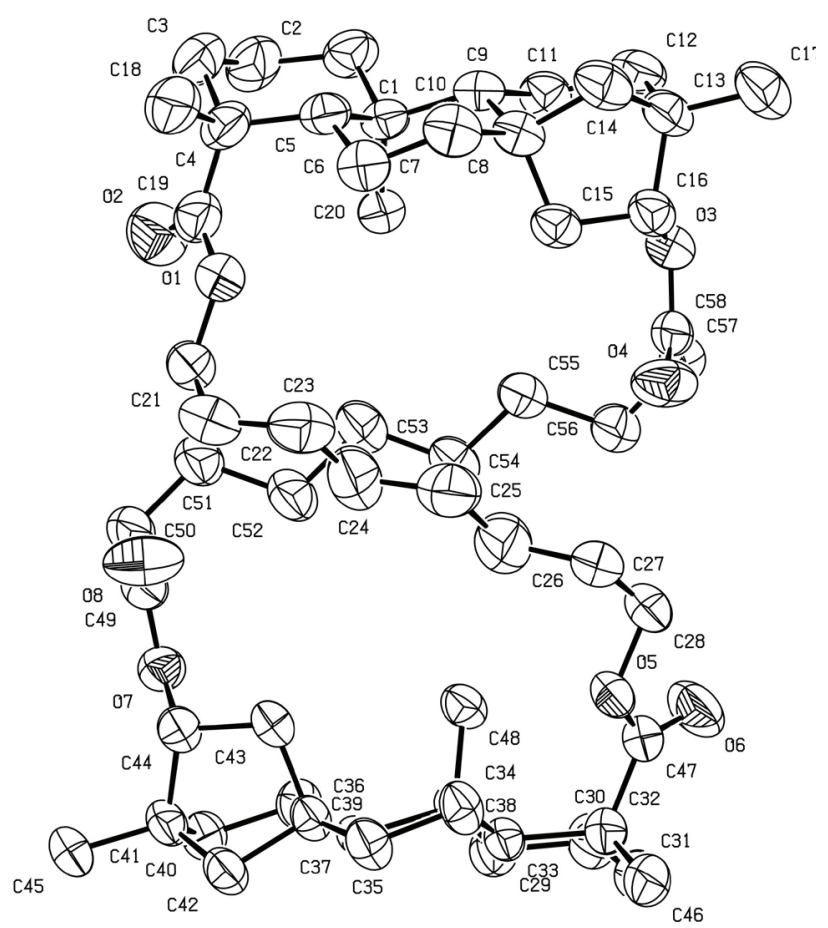

Figure 5. X-ray structure of macrocycle 13 (ORTEP drawing, hydrogen atoms are not shown).

which also had sandwich-like structure according X-ray data (Figure 7). This fact allow us to propose that either unfolded structure of diacid $\mathbf{5}$ independently turns into sandwich-like structure when going from crystal to the reaction solution or a structure reorganization occurs in the course of the reaction. The second version seems to be more probable but the fact demands a special study.

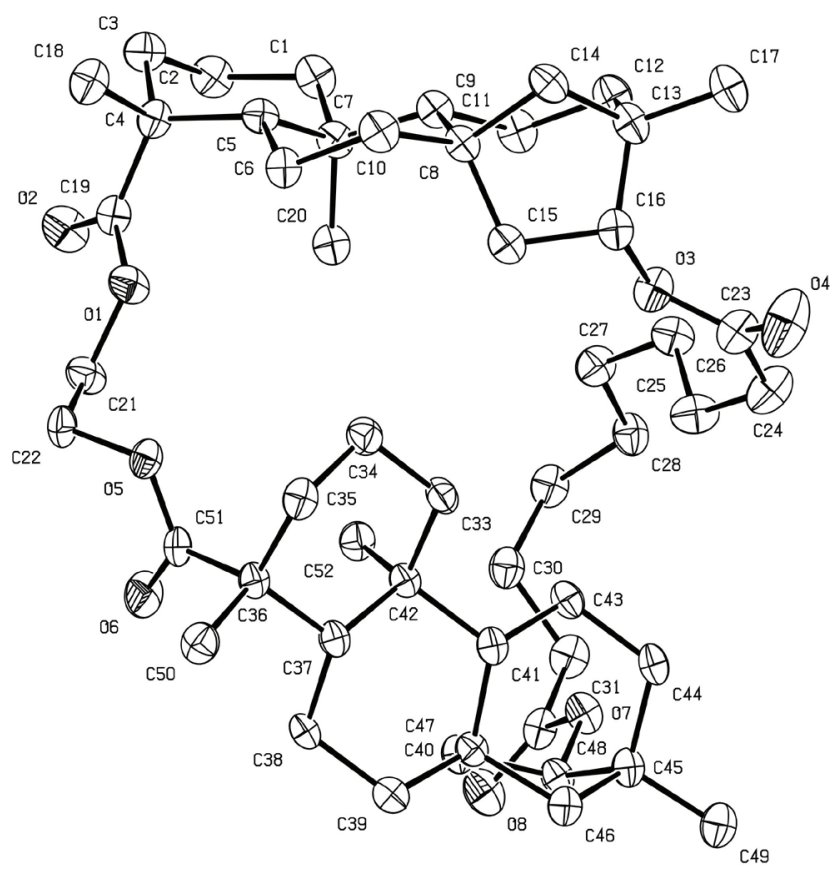

Figure 6. X-ray structure of macrocycle 15 (ORTEP drawing, hydrogen atoms are not shown).

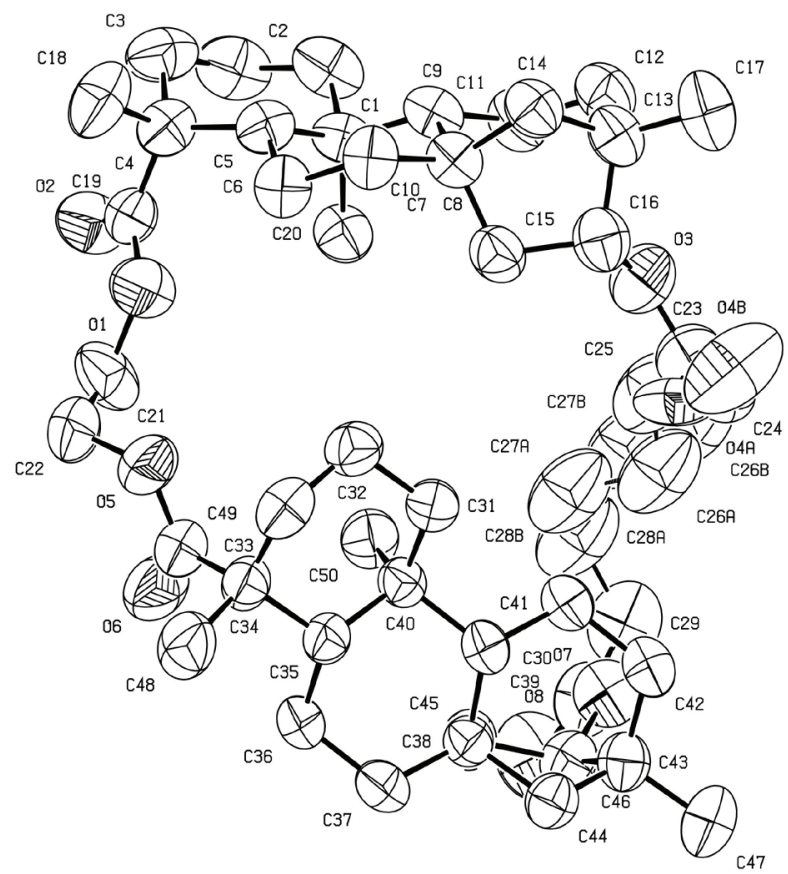

Figure 7. X-ray structure of macrocycle 9 (ORTEP drawing, hydrogen atoms are not shown).

\section{Conclusions}

A series of binuclear and tetranuclear macrocyclic derivatives of diterpenoid isosteviol were obtained with the help of routine methods of organic synthesis. Isosteviol derivatives $\mathbf{5}$ and $\mathbf{6}$ synthesised by the reaction of dihydroisosteviol $\mathbf{4}$ with dicarboxylic acids dichlorides were used as a starting 
material for macrocyclization. According to the X-ray data, the elongation of the linker connecting isosteviol moieties occurring when going from diacid $\mathbf{5}$ to diacid $\mathbf{6}$ up to only two methylene units results in the dramatic change in the molecular structure. Compound $\mathbf{5}$ has maximal unfolded molecular structure in crystal, while compound $\mathbf{6}$ has maximal folded, sandwich-like molecular structure in crystal. Both tosylate and chloro-anhydride pathways of macrocyclization led mainly to binuclear macrocycles obtained in 17-86\% yields. Exactly the sandwich-like molecular structure of starting compound $\mathbf{6}$ which is perfectly predisposed to the macrocyclization by the ditosylate of sebacic acid was the reason of the formation of binuclear mactocycle $\mathbf{1 3}$ in a very good yield (86\%). As regards to tetranuclear macrocycles, they were obtained by the macrocyclization of diacid $\mathbf{5}$ having folded molecular structure that confirmed our hypothesis. However, despite our hypothesis, the length of binucleophile used for macrocyclization was of no importance at all. The macrocyclization of diacid $\mathbf{6}$ having unfolded sandwich-like molecular structure did not afford tetranuclear macrocycle even when the most "short" binucleophile - ethylene glycol - was used. The yields of tetranuclear macrocycles 10-12 after chromatography on silica gel (2-7 \%) were rather small and practically the same. In all probability, this is caused by a statistical factor - in terms of entropy the formation of two "small" molecules of binuclear macrocycle from two molecules of diacid $\mathbf{5}$ (or 6) and two molecules of ditosylate is more preferably than the formation of one "bulky" molecule of tetranuclear macrocycle.

Acknowledgments. This work was supported by Russian Foundation for Basic Research (grant 13-03-00054-a) and the RAS Presidium Program no. 8.

\section{References}

1. a) Bridel M., Lavieille R. J. Pharm. Chim. 1931, 14, 369-379; b) Mosettig E., Nes W.R. J. Org. Chem. 1955, 20, 884-899; c) Rodrigues A.M.G.D., Lechat J.R. Acta Crystall. Sect. C 1988, 44, 1963-1965; d) Dolder F., Lichti H., Mosettig E., Quitt P. J. Am. Chem. Soc. 1960, 82, 246-247; e) Waters J.A., Becker E.D., Mosettig E. J. Org. Chem. 1962, 27, 4689-4691; f) Coates R.M., Bertram E.F. J. Org. Chem. 1971, 36, 2625-2631; g) Coates R.M., Kang H.-Y. J. Org. Chem. 1987, 52, 2065-2074; h) Hershenhorn J., Zohar M., Crammer B., Ziv Z., Weinstein V., Kleifeld Y., Lavan Y., Ikan R. Plant Growth Regul. 1997, 23, 173-178; i) Ikeda T., Kajimoto T., Kondo H., Wong C.-H. Bioorg. Med. Chem. Lett. 1997, 7, 2485-2490.

2. For a general review on isosteviol chemistry till 2013 see: a) Kataev V.E., Khaybullin R.N., Sharipova R.R., Strobykina I.Yu. Rev. J. Chem. 2011, 1, 93-160; b) Moons N., de Borggraeve W., Dehaen W. Curr. Org. Chem. 2011, 15, 2731-2741; c) Lohoelter Ch., Weckbecker M., Siegfried R.W. Eur. J. Org. Chem. 2013, 25, 5539-5554.

3. a) Ukiya M., Sawada Sh., Kikuchi T., Kushi Y., Fukatsu M., Akihisa T. Chem. Biodiversity 2013, 10, 177-183; b) Zou M., Yu S.-S., Wang K., Zhang D.-Y., Wu X.-M., Hua W.-Yi. Chin. J. Nat. Med. 2013, 11, 289-295; c) Hutt O.E., Doan T.L., Georg G.I. Org. Lett. 2013, 15, 1602-1605; d) Zhu S.-L., Wu Y., Liu
C.-J., Wei C.-Y., Tao J.-C., Liu H.-M. Eur. J. Med. Chem. 2013, 65, 70-82; e) Zhu S.-L., Wu Y., Liu C.-J., Wei C.-Y., Tao J.-C., Liu H.-M. Bioorg. Med. Chem. Lett. 2013, 23, 1343-1346; f) Ma Z.-W., Wu Y., Sun B., Du H.-L., Shi W.-M., Tao J.-C. Tetrahedron: Asymmetry 2013, 24, 7-13; g) Geng Z.-C., Chen X., Zhang J.-X., Li N., Chen J., Huang X.-F., Zhang S.-Y., Tao J.-C., Wang X.-W. Eur. J. Org. Chem. 2013, 22, 4738-4743.

4. a) Strobykina I.Yu., Garifullin B.F., Kovylyaeva G.I., Musin R.Z., Gubaidullin A.T., Kataev V.E. Russ. J. Gen. Chem. 2007, 77, 1066-1068; b) Garifullin B.F., Strobykina I.Yu., Lodochnikova O.A., Musin R.Z., Gubaidullin A.T., Kataev V.E. Chem. Natur. Compd. 2011, 47, 422-427; c) Khaybullin R.N., Strobykina I.Yu., Gubskaya V.P., Fazleeva G.M., Latypov Sh.K., Kataev V.E. Mendeleev Commun. 2011, 21, 134-136; d) Korochkina M.G., Nikitashina A.D., Khaibullin R.N., Petrov K.A., Strobykina I.Yu., Zobov V.V., Kataev V.E. Med. Chem. Commun. 2012, 3, 14491454; e) Garifullin B.F., Andreeva O.V., Strobykina I.Yu., Babaev V.M., Kataev V.E. Macroheterocycles 2013, 6, 184-191.

5. a) Breitmaier E. Terpenes. Wiley, 2006, 214 p. b) Shi Q.-W., Su X.-H., Kiyota H. Chem. Rev. 2008, 108, 4295-4327; c) Ferreira M.J.U., Duarte N., Lage H., Molnár J. Reversal of Multidrug Resistance by Macrocyclic and Polycyclic Diterpenoids from Euphorbia species, Ch. 8. In: Recent Progress in Medicinal Plants. Vol. 32. Ethnomedicine and Therapeutic Validation. (Kaushik G., Govil J.N., Eds.), Houston: Studium Press Lic., 2012, 448 p.

6. a) Bruker. Area detector control and integration software. Version 5.x. In: SMART and SAINT. Madison, Wisconsin (USA): Bruker Analytical X-ray Instruments Inc.; 1996; b) Bruker. APEX2 Software Suite for Crystallographic Programs, Bruker AXS, Inc., Madison, WI, USA, 2009; c) Sheldrick G.M., SHELX-97: Programs for Crystal Structure Analysis, University of Göttingen, Institut für Anorganische Chemie der Universität, Tammanstrasse 4, D-3400 Göttingen, Germany, 1997; d) Sheldrick G.M. Acta Cryst., A 2008, 64. 112-122; e) Farrugia L.J. WinGX and ORTEP for Windows: an update. $J$. Appl. Cryst. 2012, 45, 849-854; f) Spek A.L. J. Appl. Cryst. 2003, 36, 7-13; g) Macrae C.F., Edgington P.R., McCabe P., Pidcock E., Shields G.P., Taylor R., Towler M., van de Streek J. J. Appl. Cryst. 2006, 39, 453-457.

7. Khaibullin R.N., Strobykina I.Yu., Kataev V.E., Lodochnikova O.A., Gubaidullin A.T., Musin R.Z. Russ. J. Gen. Chem. 2009, 79, 967-971.

8. Al'fonsov V.A., Bakaleinik G.A., Gubaidullin A.T., Kataev V.E., Kovylyaeva G.I., Konovalov A.I., Litvinov I.A., Strobykina I.Y., Strobykin S.I., Andreeva O.V., Korochkina M.G. Russ. J. Gen. Chem. 2000, 70, 953-960.

9. Kataev V.E., Militsina O.I., Strobykina I.Yu., Kovylyaeva G.I., Musin R.Z., Fedorova O.V., Rusinov G.L., Zueva M.N., Mordovskoi G.G., Tolstikov A.G. Pharm. Chem. J. 2006, 40, 473475.

10. Kataev V.E., Militsina O.I., Strobykina I.Yu., Gubaidullin A.T., Zverev V.V., Kataeva O.N., Fedorova O.V., Valova M.S., Rusinov G.L. J. Incl. Phenom. 2008, 60, 51-58.

11. a) An Y., Zhang Y.-X., Wu Y., Liu Z.-M., Pi C., Tao J.-C. Tetrahedron: Asymmetry 2010, 21, 688-694; b) Pariwat P., Homvisasevongsa S., Muanprasat C., Chatsudthipong V. J. Pharm. Exp. Ther. 2008, 324, 798-805; c) Chen J., Sun M., Cai J., Cao M., Zhou W., Ji M. J. Chem. Crystallogr. 2011, 41, 519522.

12. Wu Y., Yang J.-H., Dai G.-F., Liu C.-J., Tian G.-Q., Ma W.-Y., Tao J.-C. Bioorg. Med. Chem. 2009, 17, 1464-1473.

13. Kataev V.E., Strobykin S.I., Timosheva A.P., Al'fonsov V.A., Korochkina M.G., Strobykina I.Yu. Russ. J. Gen. Chem. 2005, $75,244-247$. 